\title{
Molecular Profiling in Cutaneous Melanoma
}

\author{
Andrew L. Ji, MD ${ }^{\mathrm{a}, \mathrm{b}}$; Christopher K. Bichakjian, MDc; and Susan M. Swetter, MD ${ }^{\mathrm{a}, \mathrm{b}}$
}

\begin{abstract}
Molecular profiling of malignant tumors is gaining increasing interest in oncology. In recent years, several molecular techniques have been studied in melanoma, with the goal to improve upon the diagnostic and prognostic abilities of currently available clinical and histopathologic parameters. Reliable tests performed early in the diagnosis and management of melanoma could lead to decreased morbidity and mortality by selecting appropriate patients for more-aggressive therapy and sparing those for whom it is not indicated. This article reviews the molecular diagnostic and prognostic techniques currently available for melanoma and evaluates their potential role in clinical practice.
\end{abstract}

J Natl Compr Canc Netw 2016;14(4):475-480

The American Cancer Society estimates that approximately 76,380 cases of invasive cutaneous melanoma will be diagnosed in the United States in 2016. ${ }^{1}$ Despite recent advances in the management of patients with advanced disease through immunotherapy and targeted agents, the number of deaths in the United States from metastatic melanoma is estimated to exceed 10,000 for the first time in 2016. It therefore remains of paramount importance that additional tools are developed to aid in the early diagnosis and accurate prognostication of primary cutaneous melanoma.

Currently, the diagnosis of melanoma relies primarily on histopathologic and immunohistochemical findings. Several characteristics of the primary tumor (eg, Breslow thickness, presence or absence of ulceration, and number of dermal mitoses per $\mathrm{mm}^{2}$ ) and the presence or absence of occult nodal metastasis identified by sentinel lymph node biopsy (SLNB) are known to correlate with outcome. These prognostic parameters are currently incorporated in the AJCC staging system, which is used to risk-stratify patients and determine appropriate stage-based treatment and surveillance man-

From aVA Palo Alto Health Care System, Dermatology Service, Palo Alto, and 'Stanford University Medical Center and Cancer Institute, Department of Dermatology Department of Dermatology, Pigmented Lesion and Melanoma Program, Stanford, California; and University of Michigan Health System, Department of Dermatology, Ann Arbor, Michigan. Submitted February 5, 2016; accepted for publication March 1, 2016. agement. Several models have been developed in the United States and abroad to provide individualized prognostication for patients with localized or regional cutaneous melanoma, incorporating known prognostic parameters. ${ }^{2-8}$ However, diagnostically challenging melanocytic lesions, such as atypical Spitz tumors, remain difficult to risk stratify based on conventional criteria.

Most patients are diagnosed with early-stage melanoma, for whom current AJCC staging provides excellent prognostication (93\% 10-year survival rate for stage IA), which allows for definitive treatment with limited morbidity and appropriate follow-up. ${ }^{9}$ For clinically node-negative patients with higher-risk melanoma based on currently known criteria, in whom the risk of regional lymph node metastasis generally exceeds $10 \%$, the sentinel lymph node (SLN) status is the most powerful predictor of survival and determines the indication for additional surgery (ie, complete lymph node dissection), systemic adjuvant therapy, surveillance imaging, and frequency of clinical follow-up. However, controversy remains regarding the most appropriate criteria to perform SLNB, particularly in T1 melanoma $(\leq 1 \mathrm{~mm})$.
The authors have disclosed that they have no financial interests, arrangements, affiliations, or commercial interests with the manufacturers of any products discussed in this article or their competitors.

Correspondence: Susan M. Swetter, MD, Department of Dermatology/ Cutaneous Oncology, Stanford University Medical Center and Cancer Institute, 900 Blake Wilbur Drive, W3045, Stanford, CA 94305.

E-mail: sswetter@stanford.edu 
Ji et al

Moreover, SLN status is not perfect in predicting recurrence; the occasional occurrence of metastatic disease in patients with SLN-negative status underscores the need for additional, more discriminant prognostic indicators.

In recent years, several molecular techniques have emerged in an attempt to augment current methods in the diagnosis, prognosis, and management of melanoma, fueled by successful efforts in breast cancer and other malignancies. Comparative genomic hybridization (CGH) and fluorescence in situ hybridization (FISH) have been explored primarily to aid in the differentiation among diagnostically challenging melanocytic lesions. Gene expression profiling (GEP) has been studied both from a diagnostic and a prognostic perspective, which has led to 2 currently commercially available assays in the United States. This review summarizes some of the ongoing efforts to bring molecular techniques into clinical practice, specifically with regard to the diagnosis and prognosis of primary cutaneous melanoma. It also provides several reasonable benchmarks needed to successfully launch molecular profiling into routine clinical practice, largely based on the ongoing success in the management of breast cancer.

\section{Diagnostic Molecular Techniques}

Melanoma is characterized by high genomic instability, with copy number gains and losses found in most lesions, a feature uncommonly encountered in benign melanocytic lesions, including Spitz nevi. ${ }^{10,11}$ CGH takes advantage of this finding by analyzing copy number variations. In a study by Raskin et al, ${ }^{12}$ the largest to date using $\mathrm{CGH}$ to evaluate atypical Spitz tumors, alterations not commonly observed in conventional melanoma were reported in 7 of 16 cases. In this small study, the lack of common melanoma alterations in these borderline melanocytic lesions suggested a distinct morphologic entity. FISH has gained recent interest as an alternative molecular technique to examine copy number variation in melanocytic lesions through evaluating genetic alterations at specific loci. Using a 4-probe FISH panel, Gerami et al $^{13}$ reported relatively high sensitivity $(86.7 \%)$ and specificity (95.4\%) when distinguishing melanoma from unequivocally benign nevi. However, when evaluating diagnostically ambiguous melanocytic lesions, several studies have reported variable clinical utility of FISH. ${ }^{12,14-16}$ Although specific abnormalities (eg, homozygous 9p21 deletion) may be associated with a higher risk of clinically aggressive behavior, chromosomal numeric abnormalities alone do not appear to be predictive of outcome in histologically ambiguous melanocytic lesions.

A currently available commercial molecular diagnostic assay (myPath Melanoma test; Myriad Genetics, Inc., Salt Lake City, UT) for differentiating melanoma from benign melanocytic neoplasms is based on GEP. In contrast to sequencing assays that assess for the presence of a particular mutation as a therapeutic target (eg, BRAFV600 in metastatic melanoma) or CGH/FISH to assess for chromosomal abnormalities, GEP measures the quantified expression of a particular set of predetermined genes. The combination of these upregulated and downregulated genes comprises a gene signature that may differentiate a benign from a malignant neoplasm, or classify a particular tumor into a specific prognostic category. The myPath Melanoma gene expression signature was devised using quantitative reverse-transcription polymerase chain reaction (RT-PCR) to identify groups of genes differentially expressed between benign and malignant melanocytic lesions, including banal/common nevi (junctional, compound, intradermal), dysplastic nevi (junctional and compound), Spitz nevi, Reed nevi, blue nevi, and other subtypes, which were selected by experienced dermatopathologists from Europe and the United States. ${ }^{17}$ The training cohort consisted of 595 cases, whereas the independent validation set included 571 melanocytic lesions selected from institutions not contributing to the training cohort. From 79 candidate biomarker genes, previously observed to have differential expression in benign nevi and melanoma, 40 of the most promising biomarkers were chosen. An optimal diagnostic gene signature of 3 components was created, each with a distinct expression profile that was verified to contribute independently to the signature. The refined gene signature had a total of 24 measurements of 23 different genes. A melanoma diagnostic score was determined to differentiate melanoma from nevi in the 437-sample final validation cohort, which included standard melanoma subtypes $(n=211)$, dysplastic nevi ( $n=67$; although not graded according to degree of cytologic atypia), and conventional nevi $(n=149)$. Performance of the signature was determined by comparing the melanoma diagnostic score with the consensus histopathologic diagnosis from the dual, blinded dermatopathologist 
assessment; 17 cases were deemed histopathologically ambiguous and further adjudicated. The melanoma diagnostic score differentiated conventional melanocytic nevi from melanoma with a sensitivity of $90 \%$ and specificity of $91 \%$. Further validation of the gene expression signature (including correlation with clinical outcome) was deemed necessary by the authors and is ongoing through prospective data collection from academic dermatopathologists nationwide. ${ }^{18}$ The effectiveness of myPath Melanoma for assessing melanocytic tumors of uncertain malignant potential, including atypical Spitz tumors, has yet to be determined. To date, no head-to-head comparisons of diagnostic accuracy of melanoma have been performed between CGH/FISH and GEP.

\section{Prognostic Molecular Techniques}

Great interest exists in the identification of a GEP to aid in the prognostication of the metastatic risk of cutaneous melanoma. A decade ago, Winnepenninckx et $\mathrm{al}^{19}$ reported a profile of 254 genes whose expression was associated with distant metastasisfree survival (DMFS) in patients with primary melanoma. Thirty-three genes that were overexpressed in melanomas from patients who remained free of metastasis for 4 years were thought to be of particular interest. In 2013, Brunner et $\mathrm{al}^{20}$ identified a 9-gene signature in melanoma, associated with overall survival (OS) and DMFS survival. Interestingly, no overlap in individual genes was noted among the signatures identified in these small sample sizes of 58 and 38 patients, respectively.

A 31-gene expression assay (DecisionDx-Melanoma; Castle Biosciences, Inc., Friendswood, TX) is currently commercially available and was designed to predict the metastatic risk of AJCC stage I and II melanoma in conjunction with other standard prognostic indicators, including stage and SLN status. DecisionDx-Melanoma is currently FDA-approved as a laboratory-developed test, and the company is seeking approval for its use as a biomarker tool. The assay, which requires tissue from either the original diagnostic skin biopsy or excisional surgery specimen, measures the expression levels of 28 discriminating genes and 3 control genes to stratify a primary cutaneous melanoma into either class 1 or class 2. Class 1 tumors are designated as low risk for developing metastasis, whereas class 2 indicates high risk. ${ }^{21,22}$ The selection of genes was based on analysis of expression data from public databases, analyzing upregulated and downregulated genes in metastatic tissue compared with primary tumors in both cutaneous and uveal melanoma. The assay shares 5 genes with DecisionDx-UM, a 15gene expression assay marketed by the same company for prognostication in uveal melanoma. Through gene ontology analysis, the authors found that the DecisionDx-Melanoma assay contains genes involved in epithelial differentiation and development, as well as cell-cell junction and non-membrane-bound organelle classes. One particular gene, BAP1, which is implicated in the metastatic potential of uveal melanoma, is represented twice in the signature with probes for both $5^{\prime}$ and $3^{\prime}$ ends. The authors do not specify how or why the remaining 26 genes in DecisionDxMelanoma were selected other than based on their expression profile differences.

DecisionDx-Melanoma was developed and validated retrospectively, using a multicenter cohort of 268 cases, stage $0-I V$, from 7 independent centers. ${ }^{21}$ Most cases had at least a 5-year follow-up, unless there was a documented metastatic event before that time. Preliminary analysis of the development cohort used 107 cases, which were further expanded to a total of 164 as the training set. The remaining 104 cases were used as a validation set. The training cohort included 130 of 164 cases with AJCC stage I and II cutaneous melanoma. Interestingly, the metastatic rates were 9 of 63 patients (14\%) with stage I disease and 39 of 67 patients (58\%) with stage II disease, for a combined rate of 48 of 130 patients (37\%). This is notably higher than previously reported metastatic rates in patients with stage I-II melanoma, and suggests selection of an event-enriched training set. ${ }^{23,24}$

Analysis of the validation set predicted 61 lowrisk (class 1) samples and 43 high-risk (class 2) samples. Five-year disease-free survival (DFS) rates were 97\% for patients with class 1 tumors versus $31 \%$ with class $2(P<.001)$. OS was $100 \%$ for class 1 and $68 \%$ for class 2 ; significant differences were also found in DMFS and melanoma-specific survival. Within the validation set, comparison of GEP with other AJCC prognostic factors, including stage IIB/IIC status, Breslow thickness, ulceration, mitotic rate (dichotomized at $1 / \mathrm{mm}^{2}$ and not further stratified), and age, revealed that the GEP signature was an independent predictor of metastatic risk in both univariate and multivariate analyses; SLN status was not incorpo- 
Ji et al

rated into the multivariate model used to analyze the impact of GEP on outcome.

In a second publication, Gerami et $\mathrm{al}^{22}$ report their findings with DecisionDx-Melanoma in a validation cohort of 217 patients with cutaneous melanoma, identified as a convenience sample from a database of 406 cases previously tested with the GEP assay. The inclusion criteria and date of diagnosis range were identical to the previous study; however, all patients in the current cohort underwent SLNB. Notable differences between this cohort and previous studies ${ }^{24}$ include a higher SLN positivity rate of $27 \%$ (58 of 217), and a much greater metastatic rate of $45 \%$ among SLN-negative patients (18 of 159 patients developing nodal and 53 of 159 distant metastases). This again suggests that the convenience sample was an unusually event-enriched validation cohort. The authors report that in this cohort, GEP was comparable to SLNB in its ability to predict distant metastasis. On multivariate analysis, both GEP and SLNB were shown to be independent predictors of DFS and DMFS ( $P<.008$ for both), whereas only GEP was found to be an independent predictor for OS $(P<.0001)$. However, relevant primary tumor characteristics were not incorporated into the multivariate model in this analysis. The authors further suggest improved prognostication by combining GEP and SLNB results. However, 5-year DFS for all patients with class 1 tumors $(n=76)$ was not significantly different than for patients with class 1 tumors and a negative SLNB $(n=67)$, at $79 \%$ and $83 \%$, respectively. Moreover, 5-year DFS in patients with SLN-negative status $(\mathrm{n}=159)$ was surprisingly low $(55 \%)$, and was similar to the small group of patients $(n=9)$ with class 1 tumors and a positive SLNB $(53 \%)$. In fact, 5-year OS in the latter subset was nearly identical to that of patients with AJCC stage IIIA disease (1-3 regional nodal micrometastasis without primary tumor ulceration), at $77 \%$ vs $78 \%$, respectively. These findings suggest that patients in this particular validation set may not be representative of all patients with clinical stage I-II melanoma.

Among patients with high-risk class 2 tumors, regardless of SLN status (positive or negative), 5-year DFS was nearly identical to that of patients with a positive SLNB who were classified by GEP as having either class 1 or class 2 tumors (range, 33\%-37\%). Very similar trends were reported for DMFS and OS. Although the authors highlight the unfavorable out- come in the $42 \%$ of SLN-negative patients with a high-risk class 2 GEP result (5-year DFS, 35\%; OS, $55 \%)$, the unexpectedly high metastatic rate among patients with SLN-negative status in the entire study cohort makes this observation less significant.

The authors did not provide concurrent AJCC staging data to determine whether any of the widely accepted staging characteristics (eg, Breslow thickness, ulceration, mitotic rate) or other adverse histopathologic features (eg, lymphovascular invasion) could potentially be combined with GEP to help categorize the more challenging aforementioned class 2/SLN-negative group.

\section{Discussion}

Every clinician familiar with melanoma has encountered a patient who has developed advanced metastatic disease, despite favorable known prognostic indicators at the time of diagnosis. Primary tumor characteristics and SLN status can provide invaluable information, but many questions regarding prognostication remain unanswered. Recent retrospective studies have shown that molecular techniques may be promising diagnostic and/or prognostic tools, but significant challenges remain to validate their use in clinical practice, including the difficulty of designing and powering prospective randomized controlled trials. ${ }^{25}$

During the past decade, many of these obstacles were overcome in the development of a 21-gene GEP assay (Oncotype DX; Genomic Health, Redwood City, CA) for breast cancer management. To date, Oncotype DX is the only GEP assay included in the NCCN Clinical Practice Guidelines in Oncology (NCCN Guidelines) for Breast Cancer. ${ }^{26}$ The assay calculates a recurrence score to help determine the risk of distant recurrence in patients with node-negative, estrogen receptor-positive breast cancer, and the potential benefit of adjuvant chemotherapy. The first validation studies on Oncotype DX were conducted with a prospectiveretrospective design, using archived specimens from a previously conducted prospective trial. Clearly defined end points were established to reduce the obvious selection bias of a convenience sample set. ${ }^{27,28}$ Initial studies showed that the recurrence score could accurately quantify the likelihood of distant recurrence. ${ }^{27}$ Subsequent studies corroborated this finding and demonstrated that adjuvant chemotherapy was of greatest benefit to patients with the highest recurrence scores, while sparing 
those patients with low scores the morbidity associated with chemotherapy. ${ }^{28,29}$ The success of Oncotype DX may also stem from the narrow focus on patients with estrogen-positive, node-negative breast cancer rather than all types of breast cancer. A similarly focused approach for melanoma may prove to be useful given its high mutational burden and genetic heterogeneity.

Two recently published studies on DecisionDxMelanoma suggest that a 28-gene signature profile may accurately predict metastatic risk in patients with primary cutaneous melanoma, particularly in those undergoing concurrent SLNB. Although this may apply to the cohorts studied, the small nonrepresentative sample size with an unusually high metastatic rate, particularly among patients with SLNnegative status, limits the validity of conclusions about the clinical utility of this GEP test in all patients with clinical stage I-II melanoma. Significantly larger cohorts with more representative metastatic characteristics are needed to confirm the current findings. At present, it is unknown who should be offered GEP testing and how the information should be used in clinical practice. Prospective studies are in progress to address these clinically relevant questions. ${ }^{30}$ Currently, the NCCN Guidelines for Melanoma do not recommend GEP at baseline (before or following SLNB) outside of a clinical study or trial. ${ }^{31}$

In order for an assay to be of practical clinical use, reliability and reproducibility are among the most important factors to consider. In the case of GEP, the genes that comprise an assay should be selected based on an established association with the disease in question across independent samples before undergoing prospective studies. ${ }^{27}$ Although both myPath Melanoma and DecisionDx-Melanoma used similar methodologies to identify candidate genes, there were notable differences in the selection of genes for the final assay. For myPath Melanoma, the authors selected genes with the highest differentiating features and added others to improve assay reliability, an approach that takes the above factors into account. For DecisionDx-Melanoma, selection was based on differences in gene expression between metastatic and primary tissue in both cutaneous and uveal melanoma, disease entities with limited biological similarity. ${ }^{32}$ The rationale for this approach is not sufficiently explained by the authors. Finally, the noticeable lack of candidate gene overlap among DecisionDx-Melanoma and previously published prognostic gene signatures highlights the challenge of identifying the most relevant gene expression differences in such a genetically heterogenous disease. ${ }^{19,20,33}$

If GEP is demonstrated to be an accurate, standalone, prognostic method to identify patients with SLN-negative status at high risk for metastasis, it could serve as a key biomarker to identify patients who should undergo complete lymph node dissection following a positive SLNB and/or those who should be treated with systemic adjuvant therapy. Ideally, GEP could obviate the need for SLNB entirely and determine the metastatic potential of primary cutaneous melanoma at the time of diagnosis. As more effective and tolerable systemic therapies become available in the adjuvant setting for patients with high-risk cutaneous melanoma, GEP could prove to be the preferred molecular technique. Currently, a limited number of adjuvant therapy options are available for patients with resected high-risk melanoma, including alfa interferon (given as highdose for 1 year or as pegylated interferon alfa-2b for up to 5 years), biochemotherapy, and high-dose ipilimum$\mathrm{ab}^{34-37}$ Although these regimens have shown improved relapse-free survival with no substantial improvement in OS, they are associated with significant side effects and potential toxicities, limiting their use in clinical practice. The addition of GEP as a biomarker tool could help determine patient eligibility for currently available adjuvant therapies and guide future trials.

Finally, molecular diagnostic techniques using $\mathrm{CGH}$, FISH, or GEP appear to demonstrate promising results in the differentiation between histopathologically unequivocal benign and malignant melanocytic lesions. However, until sensitivity and specificity in the diagnosis of histopathologically equivocal lesions of uncertain biological behavior is demonstrated, the clinical utility of molecular techniques for diagnosis of cutaneous melanoma is limited.

\section{Conclusions}

In an era of extraordinary changes in oncology, molecular profiling is emerging as an exciting and highly promising tool to diagnose a primary malignancy and predict its metastatic potential. Molecular techniques could be used to differentiate our most challenging diagnostic cases and to identify patients, at the time of diagnosis, who are in need of more extensive surgery and/or systemic adjuvant therapy. 
Ji et al

Equally important, a reliable biomarker could spare many patients the unnecessary morbidity, anxiety, and cost associated with cancer therapy and ongoing surveillance. Although significant advances have been made with other malignancies, GEP has yet to be fully validated in cutaneous melanoma and requires rigorous evaluation in the context of all known clinicopathologic variables before it is accepted for routine risk assessment. Identification of the most relevant candidate genes, standardized RT-PCR techniques, and well-defined criteria for overexpression and underexpression of these genes are critical to support these tests as standard practice. Larger studies, preferably using the prospectiveretrospective design successfully applied in breast cancer, are needed to confirm the current preliminary findings and to define the clinical utility of GEP in patients with primary cutaneous melanoma.

\section{References}

1. American Cancer Society. Cancer Facts \& Figures 2016. Available at: http://www.cancer.org/acs/groups/content/@research/documents/ document/acspc-047079.pdf. Accessed march 14, 2016

2. Soong S, Ding S, Coit DG, et al. AJCC Individualized Melanoma Patient Outcome Prediction Tools. Available at: http://melanomaprognosis.org/. Accessed March 14, 2016.

3. Callender GG, Gershenwald JE, Egger ME, et al. A novel and accurate computer model of melanoma prognosis for patients staged by sentinel lymph node biopsy: comparison with the American Joint Committee on Cancer model. J Am Coll Surg 2012;214:608-617.

4. Baade PD, Royston $P$, Youl PH, et al. Prognostic survival model for people diagnosed with invasive cutaneous melanoma. BMC Cancer 2015;15:27.

5. Fears TR, Guerry D IV, Pfeiffer RM, et al. Identifying individuals at high risk of melanoma: a practical predictor of absolute risk. J Clin Oncol 2006;24:3590-3596.

6. Melanoma Risk Assessment Tool. National Cancer Institute Web site. Available at: http://www.cancer.gov/melanomarisktool/. Accessed March 14, 2016.

7. Melanoma Prediction Tools. Memorial Sloan Kettering Cancer Center Web site. Available at: https://www.mskcc.org/cancer-care/types/ melanoma/prediction-tools. Accessed March 14, 2016.

8. CancerMath.net Melanoma Outcome Calculator. Available at: http:// www.lifemath.net/cancer/melanoma/outcome/. Accessed March 14, 2016.

9. Balch CM, Gershenwald JE, Soong SJ, et al. Final version of 2009 AJCC melanoma staging and classification. J Clin Oncol 2009;27:6199-6206.

10. Martinocorena I, Campbell PJ. Somatic mutation in cancer and normal cells. Science 2015;349:1483-1489.

11. Bastian $B C$, Olshen $A B$, LeBoit PE, Pinkel D. Classifying melanocytic tumors based on DNA copy number changes. Am J Pathol 2003;163:17651770.

12. Raskin L, Ludgate M, Iyer RK, et al. Copy number variations and clinical outcome in atypical spitz tumors. Am J Surg Pathol 2011;35:243-252.

13. Gerami P, Jewell SS, Morrison LE, et al. Fluorescence in situ hybridization (FISH) as an ancillary diagnostic tool in the diagnosis of melanoma. Am J Surg Pathol 2009;33:1146-1156.

14. Gaiser $T$, Kutzner H, Palmedo G, et al. Classifying ambiguous melanocytic lesions with FISH and correlation with clinical long-term follow up. Mod Pathol 2010;23:413-419.

15. Vergier B, Prochazkova-Carlotti M, de la Fouchardière, et al. Fluorescence in situ hybridization, a diagnostic aid in ambiguous melanocytic tumors: European study of 113 cases. Mod Pathol 2011;24:613-623.
16. Gerami P, Li G, Pouryazdanparast P, et al. A highly specific and discriminatory FISH assay for distinguishing between benign and malignant melanocytic neoplasms. Am J Surg Pathol 2012;36:808-817.

17. Clarke LE, Warf BM, Flake DD II, et al. Clinical validation of a gene expression signature that differentiates benign nevi from malignant melanoma. J Cutan Pathol 2015;42:244-252.

18. Rock C, Cockerell C, Tschen J, et al. A retrospective study of the influence of a gene expression signature on the treatment of melanocytic tumors. Poster presented at The 52nd Annual Meeting of the American Society of Dermatopathology; October 8-11, 2015; San Francisco, California. Number 457

19. Winnepenninckx V, Lazar V, Michiels S, et al. Gene expression profiling of primary cutaneous melanoma and clinical outcome. J Natl Cancer Inst 2006;98:472-482.

20. Brunner $G$, Reitz $M$, Heinecke $A$, et al. A nine-gene signature predicting clinical outcome in cutaneous melanoma. J Cancer Res Clin Oncol 2013;139:249-258.

21. Gerami P, Cook RW, Wilkinson J, et al. Development of a prognostic genetic signature to predict the metastatic risk associated with cutaneous melanoma. Clin Cancer Res 2015;21:175-183.

22. Gerami P, Cook RW, Russell MC, et al. Gene expression profiling for molecular staging of cutaneous melanoma in patients undergoing sentinel lymph node biopsy. J Am Acad Dermatol 2015;72:780-785.

23. Gershenwald JE, Colome MI, Lee JE, et al. Patterns of recurrence following a negative sentinel lymph node biopsy in 243 patients with stage I or II melanoma. J Clin Oncol 1998;16:2253-2260.

24. Morton DL, Thompson JF, Cochran AJ, et al. Final trial report of sentinelnode biopsy versus nodal observation in melanoma. N Engl J Med 2014;370:599-609.

25. Simon RM, Paik S, Hayes DF. Use of archived specimens in evaluation of prognostic and predictive biomarkers. J Natl Cancer Inst 2009;101:14461452 .

26. Gradishar WJ, Anderson BO, Balassanian R, et al. NCCN Clinical Practice Guidelines in Oncology: Breast Cancer. Version 1.2016. Accessed March 1, 2016. To view the most recent version of these guidelines, visit NCCN.org.

27. Paik S, Shak S, Tang G, et al. A multigene assay to predict recurrence of tamoxifen-treated, node-negative breast cancer. N Engl J Med 2004;351:2817-2826.

28. Paik S, Tang G, Shak S, et al. Gene expression and benefit of chemotherapy in women with node-negative, estrogen receptor-positive breast cancer. J Clin Oncol 2006;24:3726-3734

29. Sparano JA, Gray RJ, Makower DF, et al. Prospective validation of a 21gene expression assay in breast cancer. N Engl J Med 2015;373:2005-2014

30. Hsueh EC, Laks S, Hunborg PS, et al. Gene expression profile test adds prognostic information in management of primary melanoma. Presented at the Society of Surgical Oncology 68th Annual Cancer Symposium; March 25-28, 2015; Houston, Texas. Abstract P234.

31. Coit DG, Thompson JA, Algazi A, et al. Clinical Practice Guidelines in Oncology: Melanoma. Version 2.2016. Accessed March 1, 2016. To view the most recent version of these guidelines, visit NCCN.org.

32. Luke JJ, Triozzi PL, McKenna KC, et al. Biology of advanced uveal melanoma and next steps for clinical therapeutics. Pigment Cell Melanoma Res 2015;28:135-147.

33. Nsengimana J, Laye J, Filia A, et al. Independent replication of a melanoma subtype gene signature and evaluation of its prognostic value and biological correlates in a population cohort. Oncotarget 2015;6:11683-11693.

34. Eggermont AM, Suciu S, Testori A, et al. Long-term results of the randomized phase III trial EORTC 18991 of adjuvant therapy with pegylated interferon alfa-2b versus observation in resected stage III melanoma. J Clin Oncol 2012;30:3810-3818.

35. Eggermont AM, Chiarion-Sileni V, Grob JJ, et al. Adjuvant ipilimumab versus placebo after complete resection of high-risk stage III melanoma (EORTC 18071): a randomised, double-blind, phase 3 trial. Lancet Oncol 2015;2045:522-530.

36. Flaherty LE, Othus M, Atkins MB, et al. Southwest Oncology Group S0008: a phase III trial of high-dose interferon Alfa-2b versus cisplatin, vinblastine, and dacarbazine, plus interleukin-2 and interferon in patients with high-risk melanoma-an intergroup study of Cancer and Leukemia Group B, Children's Oncology Group, Eastern Cooperative Oncology Group, and Southwest Oncology Group. J Clin Oncol 2014;32:3771-3778.

37. Rafique I, Kirkwood JM, Tarhini AA. Immune checkpoint blockade and interferon- $\alpha$ in melanoma. Semin Oncol 2015;42:436-447. 